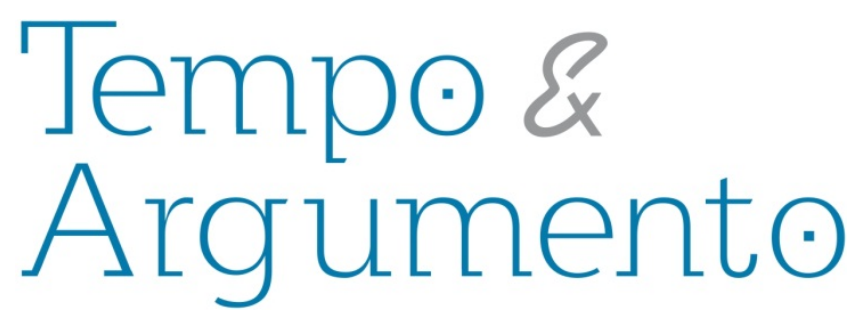

\title{
Dossier Bergery : une trajectoire politique dans I'histoire récente de la France
}

\begin{abstract}
Résumé
Gaston Bergery, né en 1892 et mort en 1974, était un avocat et un homme politique français. D'éditeur de la revue politique La Flèche il est devenu ambassadeur en Turquie durant le gouvernement de Vichy. Nous essayons, à travers l'analyse de lettres, articles et d'autres documents personnels et publics, de tracer la trajectoire de cet individu politiquement actif au cours de la première moitié du XXe siècle. Pour cela nous avons fait des recherches approfondies dans plusieurs archives, y comprises celles du Château de Vincennes (archives de l'Armée), du Quais d'Orsay (archives duministère des Affaires étrangères), des Archives nationales, de la Fondation Hoover, de l'Université d'Yale, ainsi que des entretiens oraux.
\end{abstract}

Mots-clés : Archives. Régime de Vichy. Diplomatie. Gaston Bergery.
Yves Pourcher

Professeur à l'Université de

Toulouse Le Mirail.

yves.pourcher@sciencespo-toulouse.fr

\section{Pour citer cet article :}

POURCHER, Yves. Dossier Bergery : une trajectoire politique dans l'histoire récente de la

France. Revista Tempo e Argumento. Florianópolis, v. 5, n.9, jan./jun. 2013. p. 378 - 404. 


\section{The Bergery file: a political trajectory in the recent history of France}

\begin{abstract}
Gaston Bergery was born in 1892 and died in 1974. He was a lawyer and a French politician. From editor of the political magazine La Flèche, he became the ambassador in Turkey during the Vichy government. We aim to, through the analysis of letters, articles and other personal and public documents, trace the trajectory of this individual, who was politically active during the first half of the twentieth century. To this, we have made an extensive research through several files, including that of the Castle of Vincennes (the Army file), the Quais d'Orsay (the Ministry of Foreign Affairs files), the National Archives, the Hoover Foundation, Yale University, as well as some oral interviews.
\end{abstract}

Keywords: Archives. Vichy Regime. Diplomacy. Gaston Bergery.

\section{Pasta Bergery: uma trajetória política na história recente da frança}

\begin{abstract}
Resumo
Gaston Bergery, nascido em 1892 e falecido em 1974, foi advogado e político francês. De editor de política da revista La Flèche a embaixador na Turquia durante o governo de Vichy. Busca-se, através da análise de cartas, artigos e outros documentos pessoais e públicos, traçar a trajetória deste indivíduo politicamente atuante durante a primeira metade do século XX. Para isto foi feita uma extensa pesquisa por diversos arquivos entre os quais 0 do Castelo de Vincennes (arquivo do exército), do Cais de Orsay (arquivos do Ministério dos Assuntos Estrangeiros), os Arquivos Nacionais, da fundação Hoover, da Universidade de Yale, bem como algumas entrevistas orais.
\end{abstract}

Palavras-chaves: Arquivos. Regime de Vichy. Diplomacia. Gaston Bergery. 
C'est l'idée du passage qui s'était peu à peu imposée. À peine une porte se fermaitelle qu'une autre s'ouvrait aussitôt avec un nouveau chemin que je devais absolument parcourir. En disant cela, je ne recours pas à une facile évocation poétique et je ne me lance surtout pas dans une recomposition empreinte de nostalgie. Cette errance, je l'ai vécue, et difficilement. Sans cesse en avançant, je ressentais cette impossibilité à atteindre le but fixé. Je dois bien avouer aujourd'hui et reconnaître ce qui n'est pas tout à fait un échec, mais une course laborieuse semée d’obstacles. Tout cela pour tenter d'avancer dans l'histoire à la poursuite d'un personnage oublié. À propos de lui, j'ai essentiellement gardé une succession de lieux où j'amassais des rapports, des lettres, des discours et des photos. Pour composer les contours tremblants de son portrait, j'ai volontairement choisi d'en retenir des extraits en les recopiant. Mieux que tout autre commentaire ou analyse, ils révèlent l'ambiguïté du personnage et le trouble de l'époque qu'il a traversée. Désordre des idées, paradoxe des situations avec des croyants et des renégats, et puis cet assourdissant bruit de bottes qui annonçait la guerre. Des images défilaient. Elles retentissaient dans ma tête.

Au tout début, j'avais commencé avec une définition générale des archives. "Collection de pièces, titres, documents, dossiers anciens », était-il marqué dans mon dictionnaire. D'elle, je retenais cette idée d'un temps passé, dissimulé, enfoui et, peutêtre, complètement oublié. Et puis, très vite, avait surgi cet intérêt pour leur couleur. Archives diurnes, nocturnes, celles du clair obscur, du gris plutôt que du blanc ?

Le sombre s'était imposé. À quoi correspondait-il ? Je cherchais son sens. «Qui est peu éclairé, reçoit peu de lumière ( $V$. Noir, obscur), est-il écrit. Qui est mêlé de noir, ou se rapproche du noir. Couleur, teinte sombre. »

Je continuais et trouvais des nuances: «Dont les pensées, les sentiments sont empreints de tristesse, d'abattement, de douleur ou d'inquiétude. V. Amer, morne, morose, taciturne, ténébreux, triste.

D’une tristesse tragique ou menaçante. V. Sinistre. V. Atrabilaire, bilieux, mélancolique, pessimiste.

D’une tristesse tragique ou menaçante. V. Funèbre, funeste, inquiétant, sinistre, 
tragique.

Déplorable, lamentable. »

Cela résonnait comme un jugement. « Il a été lamentable ! » entendais-je en écho. Alors je fouillais dans ma galerie de portraits et j'en croisais plusieurs de ces sombres individus qui étaient apparus dans l'entre-deux-guerres et avaient sombré pendant l'Occupation. Parmi eux, je cherchais un personnage de haut vol avec une magnifique carrière promise, annoncée, et puis un naufrage total et définitif. Je le trouvais enfin et suivais les jeux de lumière qui peu à peu s'imprimaient sur ses pas. Une silhouette se dessinait, hier éclairée, aujourd'hui éteinte et même rejetée. Pour raconter sa vie, je devais explorer les lieux où étaient disséminées les traces de ses gestes et de ses mots. Rien que des bouts, et des morceaux tronqués.

Je me rendis à plusieurs adresses. J'entrais, m'asseyais, ouvrais des cartons. Dans des liasses de documents, je trouvais la mise en lumière, l'ambition, le succès. Alors je m'obstinais et poursuivais. La nuit tombait, rapide, brutale. Failles, échec, compromission, faillite et mort : tout était là. L'archive murmurait.

Il avait commencé par être celui à qui tous les espoirs étaient permis, l'homme qui vient, qu'on attend et admire avant de devenir celui qui tombe, et dont il ne reste plus, et seulement pour de rares témoins, que le souvenir du passage, celui du météore. Hier pourtant, pendant toutes ces années, il avait tant brillé.

Pour bien parler de lui, je lisais des livres et fouillais une nouvelle fois ces strates d'archives qui, inexorablement, se mêlaient et se superposaient. C'était l'archive mine, dispersée, émiettée et coloriée. Celle-là, je la trouvais et j'en exhumais des bribes. Mais je voyais aussi qu'il y avait tout le reste, l’archive cachée, éloignée, réservée, fermée. Celle que je n'atteignais pas et que je n'atteindrai peut-être jamais. Alors par peur d'être incomplet, je m'éloignais et me tournais vers une autre direction. J'explorais pour tenter une fois nouvelle de m'approcher de lui et dire enfin qui il avait été. Je cherchais des indices sur sa personnalité. Plutôt que d'accumuler et lire des documents neutres ou distants, je voulais très vite le cerner, le voir, écouter les rares et précieux témoins qui subsistaient. 


\section{Esquisse biographique.}

Qui donc se souvient de lui ? Sa vie semble définitivement perdue. Il fait partie de ce petit groupe d'hommes brillants, auxquels certains ont tant cru et qui ont chu dans l'oubli de l'histoire. Celui que j'avais choisi n'était connu que de quelques historiens. Son nom, Gaston Bergery. Le résumé de sa carrière s'inscrit brièvement sur une fiche. Par exemple, celle qu'il avait constituée pour l'édition 1973-1974 du Who's Who. C'étaient les lignes qu’il avait écrites avec les mots qu’il avait choisis pour se présenter.

Je les recopie en partie:

«Bergery (Gaston, Frank) avocat honoraire, ancien député. Né le 22 novembre 1892 à Paris. Divorcé de Mme, née Germaine Malançon puis de Mme, née Lubov Krassine (fille de Leonid Krassine (1870-1926) Ambassadeur de l'URSS en France) (1 enfant : JeanFrançois); remarié le 5 août 1934 à Mlle Elisabeth-Charlotte Shaw-Jones. Études: Lycée Carnot et Faculté des lettres de Paris, Ecole de droit.

Carrière : Secrétariat de la conférence de la Paix (1918), Secrétaire général adjoint interallié à la Commission des réparations (1918-1924), Chef de cabinet du ministre des Affaires Étrangères (1924-1925), Député de Seine-et-Oise (1928-1934 et 1936-1940), Conseiller général de Seine-et-Oise (1928-1942), Ambassadeur à Moscou (1941) puis à Ankara (1942-1944); a repris (1947) sa place au barreau de Paris, avec la même spécialisation dans le droit international. Avocat honoraire (depuis 1965).

CEuvres : articles divers dans la presse ; a assuré notamment la rubrique politique de l'hebdomadaire La Flèche (1934-1939), la Libre chronique diplomatique de Paris-Presse (1957-1958). Deux ouvrages : Air Afrique, Voie impériale (1937) et (sous le pseudonyme de Gaston François) La Vierge et le Sagittaire (1948). »

Il avait reçu plusieurs décorations.

Son adresse : 29 , rue de Bourgogne, Paris $\left(7^{\mathrm{e}}\right)$.

Pour aller plus loin que ce bref exposé, je cherchais et trouvais un livre qui revenait amplement sur son parcours. Son auteur Philippe Burrin, un historien suisse spécialiste de 
la période. Le titre de son ouvrage : La dérive fasciste. Doriot, Déat, Bergery 1933-1945².

Burrin complétait utilement les données de l'annuaire des puissants. Ainsi j'apprenais que pendant la guerre 1914-1918, Gaston Bergery avait été blessé sur le front de Champagne. Après ce conflit, il avait voyagé pour affaires à Londres et Istanbul. Il avait visité aussi l'Union soviétique et les Etats-Unis. Il s'était marié trois fois, avait fréquenté les salons et les surréalistes en compagnie d'Emmanuel Berl et de Pierre Drieu La Rochelle. Ce dandy froid, provocant et solitaire, était aussi un homme de parti actif et terriblement sérieux. II voulait par-dessus tout réussir. Ses contemporains avaient souligné sa froideur, son ironie et son cynisme. Pour servir sa cause et ses intérêts politique, il avait créé un journal, La Flèche, dont le tirage se situait, en 1937, entre 25000 et 30000 exemplaires. Après la défaite de 1940, il s'était mis au service du maréchal Pétain. Bergery espérait alors prendre le pouvoir. Au mois de février 1949, il avait été jugé par la Cour de Justice de la Seine. Son procès fut d'ailleurs le seul intenté à un ambassadeur de Vichy.

Tout était là, dit en quelques lignes: l'ambition, l'attente, la chute. Mais mon tableau manquait de chair et de couleurs. Je souhaitais aller plus loin. Qui donc était Gaston Bergery ? Comment l'avaient vu ceux qui l'avaient approché ?

\section{L’ambitieux.}

Le premier que je veux citer est l'écrivain journaliste Maurice Martin du Gard. Dans le numéro des mois de juillet et août 1936 de La Revue de Paris, il avait composé un portrait qui soulignait l'allure, le tempérament, la nouveauté, le paradoxe Bergery. Pour lui, cet homme était un mystère, une inconnue, presque un défi. « C'est peu de dire, écritil, que Gaston Bergery ne rassure pas. Il s'en moque. On s'y fera, doit-il penser, car sa confiance en lui est grande, mais son esprit n'est pas petit, comme c'est parfois le lot des orgueilleux. »

\footnotetext{
${ }^{1}$ Philippe Burrin, La dérive fasciste. Doriot, Déat, Bergery 1933-1945 (Paris : Seuil, 1986). Voir aussi de Diane N. Labrosse, " "La Dérive Bergery/The Bergery Drift": Gaston Bergery and the Politics of Late Third Republic France and the Early Vichy State », Historical Reflections 34, 2 (2008), 66-87.
} 
Bergery aimait la vitesse, la musique, la peinture et le linge le plus délicat. « On se demande parfois, ajoute Martin du Gard, si Gaston Bergery est un homme d'avenir. Je pourrais répondre qu'il l'est même un peu trop, car il est toujours en avance d'une idée et d'un fait, d'un fait qui compte au moins dans la vie internationale. » Puis il avait conclu : «Il flotte autour de cette figure je ne sais quoi de dramatique et de mystérieux. Dans le veston d'une bonne étoffe bleue qui, croisé presque jusqu'au cou, laisse à peine voir le linge, les cheveux coupés courts, la parole tranchante, certes, ce n'est pas un jeune premier de comédie. Il est là, mince et droit, parmi ses livres. »

Pour Martin du Gard, Gaston Bergery attendait son heure. À côté des notables trop bien nourris du grand parti radical auquel il avait longtemps appartenu, il apparaissait déjà comme un homme nouveau en politique. Maigre, sportif, lançant des phrases courtes, il était pour certains «l'espoir de notre génération » ainsi que l'a écrit Bertrand de Jouvenel, autre journaliste engagé qui, lui aussi, sombrera dans la politique de Collaboration. Ce dernier avait également noté l'allure du personnage, ses vêtements de sport, la distinction de ce simple lacet de cuir natté autour du cou. Face à la terrible incertitude de ces années d'entre-deux-guerres, Bergery était «l'homme qui vient »".

Comme d'autres qui l'ont précédé ou qui le suivront - ainsi le jeune François Mitterrand pour l'écrivain François Mauriac - Gaston Bergery était un vrai personnage de roman. Il servit d'ailleurs de modèle à un romancier qui, lui aussi, se compromettra pendant les années noires de l'Occupation. Sous la plume de Drieu la Rochelle ${ }^{3}$, Bergery devient cet ambitieux nommé Clérences dont la volonté d'arriver semble inscrite dans les gènes. "Pendant tout le dîner, écrivait Drieu" ${ }^{4}$ il parla de l'ambition comme d'une chose qui allait de soi et qui mettait lui, et sans doute Gilles, au-dessus de la plupart des contingences humaines. » Ce dénommé Clérences incarnait déjà beaucoup d'espoirs. « Gilles méditait sur la carrière et le caractère de Clérences, racontait Drieu. N’avait-il pas assez de qualités diverses pour devenir l'homme qui concentrerait les forces des hommes

\footnotetext{
${ }^{2}$ Bertrand de Jouvenel, Un voyageur dans le siècle (Paris : Robert Laffont, 1979).

${ }^{3}$ Pierre Drieu La Rochelle, Gilles (Paris : Gallimard, 1939).

${ }^{4}$ Drieu La Rochelle, Gilles, 178.
} 
de leur âge ${ }^{5}$ ? » «As-tu l'étoffe d'un apôtre? » lui avait-il enfin demandé. L'histoire s'accélérait. Elle allait devenir folle. Au bord de sa route, Bergery trépignait.

\section{Jeunes fidèles.}

Ils sont plusieurs à se souvenir de lui parlant avec un brin de nostalgie de leurs rencontres. Fils d'écrivain prestigieux, et lui-même écrivain, Claude Mauriac a raconté dans ses mémoires l'importance qu'avait eue pour lui ce personnage. Il était alors un tout jeune homme adhérent du frontisme, mouvement et pensée politiques de Bergery qui, dans ces temps de périls, rejetait les deux extrêmes, communisme et fascisme. Pour bien comprendre la force et la séduction de cette pensée et de celui qui l'incarnait, il faut longuement citer Mauriac qui, dans son immense œuvre du Temps immobile, ne cesse de fouiller le passé. Il étire, disperse le temps glanant ici et là des moments qu'il enchaîne. Arbitrairement, je reconstruis la chronologie de ses rapports avec Bergery. Leur histoire commune avait commencé avant la guerre.

Le dimanche 4 décembre 1938, à Paris, Mauriac était allé à une réunion politique du mouvement: "Chez Vianet, quai de la Rapée, banquet de La Flèche. Trois cents personnes environ. (... ) Discours de Bergery, que les postes d'État retransmettent. De ma place, je le vois bien, assez pâle dans son éternel costume noir (col blanc, cravate noire). II lit, et le papier tremble dans ses mains. Il a l'air ému. II n'a pas, c'est visible, l'expérience du micro. Et puis la sensation d'être écouté par des Français qui ne le connaissent pas l'intimide. Il aimerait tant les gagner à sa cause par le prestige de sa voix. Jean Maze parle ensuite, mais il ne se révèle pas grand orateur. Son organe, trop faible, ne porte pas. Aussi bien l'écoute-t-on avec moins d'attention. Puis Bergery se lève une fois encore, sans papier cette fois et sans micro, et c'est beaucoup plus émouvant. Des paroles vivantes. Ce dont je ne le croyais pas capable ${ }^{6}$.»

Deux jours plus tard, le 6 décembre 1938, Mauriac se rend au numéro 29 de la rue de Bourgogne, chez Bergery où il était attendu: "Le costume noir serait-il une

\footnotetext{
${ }^{5}$ Drieu La Rochelle, Gilles, 535.

${ }^{6}$ Claude Mauriac, Le Temps Immobile 6. Le rire des pères dans les yeux des enfants (Paris : Grasset, 1981 ), 35.
} 
coquetterie politique, le discret équivalent d'un quelconque uniforme? Bergery m’apparaît pour la première fois en complet marron, cravate rouge... »

Mauriac avait regardé et écouté celui en qui il croyait tout en gardant son sens critique : «Bergery dont l'intelligence est tellement aiguë se laisse pourtant aller parfois à certaines naïvetés. Ainsi lorsqu'il me parla cet après-midi de l'étonnant effet que produisit le discours qu'il fit au micro, à la suite du banquet de l'autre soir : " Les applaudissements et les acclamations semblaient naître d'une foule immense. Cela ressemblait, paraît-il, à la rumeur du Sport-Palace." C'est sans la moindre ironie qu’il évoque les fastes hitlériens ! II se laisse prendre lui aussi aux illusions de la T.S.F ${ }^{7}{ }^{\prime}$

Après la défaite, Bergery se compromet définitivement s'excluant de l'histoire et devenant une ombre. Mauriac le revoit bien plus tard, à Paris, le lundi 29 juin 1953. "Dans la foule d'un cocktail, raconte-t-il, chez Marie-Blanche de Polignac, cet homme usé, que seule sa chemise bleue fait remarquer, Gaston Bergery, tout prestige effacé, homme sans avenir, avec son seul passé manqué. Une vieille tendresse en moi pour lui, et qui me le fait écouter avec respect, au grand étonnement de ma femme, disant avec la dureté de la jeunesse: - Son passé, on s'en fout... Pas moi, pas moi, dans la mesure où ce passé fut aussi celui de ma génération - et le mien, le plus personnel. II parle avec le brillant d'autrefois - mais qui ne brille plus. Il y fallait la nuit que faisait autour de lui sa légende. Restent des paradoxes dont la gratuité, l'inutilité sont seules sensibles. Reste un homme qui me dit sa lassitude: il est accablé de travail (au Palais), mais d'un travail qui ne l'intéresse pas... Et en effet, pour quelqu'un qui se voulait (et aurait pu être) à la tête des affaires... »

Quelques jours plus tard, le dimanche 5 juillet 1953, Mauriac dîne chez Bergery. Le regard qu'il porte alors sur cet homme, qui avait incarné tous ses espoirs, est à présent terrible : «On le sent amer, ne se consolant pas d'avoir mal joué, mais se solidarisant avec son échec, l'assumant, transformant son humiliation en orgueil et disant un peu plus souvent qu'il ne faudrait : - Moi qui suis vichyste. Présent inattendu. Mais le temps s'est peut-être arrêté pour lui à l'époque où il pouvait encore agir sur le destin des autres et sur

\footnotetext{
${ }^{7}$ Mauriac, Le Temps Immobile 6. Le rire des pères dans les yeux des enfants, 38-39.
} 
le sien propre. Du reste toujours charmant, ou plutôt séduisant, de la même façon énigmatique qui avait autrefois tant de prestige sur moi. (...)

Tandis qu'il parle, plaidant sans en avoir l'air (ce qui fait qu'il en a justement l'air) pour lui-même, je le revois, entre deux gendarmes, devant ses juges, le jour où j'allai témoigner pour lui... II m'interroge sur de Gaulle, avec passion, me faisant redire que je le trouve d'une intelligence hors du commun, exigeant de moi des détails sur sa façon d'être. Sa femme, tout aussi curieuse, essaye de me faire aller plus avant encore dans la confidence. Et voici le Bergery intelligent que j'ai bien connu. Celui dont les démonstrations semblent immédiatement convaincantes. Mais le déroulement de l'Histoire n'obéit pas à une logique aussi rigoureuse, si bien qu'il se trompe souvent (sinon toujours). »

Ce soir-là, Bergery se laisse aller aux confidences parlant longuement de cet immense pays qui, hier, l'avait tant fasciné. «Lorsque j’ai appris que les Soviets relâchaient si peu que ce soit leur étreinte, avait-il dit, j'ai pensé que le régime ne pourrait supporter cet adoucissement, si léger soit-il. J'ai été deux fois en U.R.S.S. et en des temps assez éloignés l'un de l'autre pour avoir fait les corrections nécessaires. Eh bien! ce régime ne peut tenir que par une totale contrainte. Tout se passe comme si la démocratie ne nous faisait pas de mal, (ou nous faisait qu'un mal relatif) parce que notre organisme y est habitué, si bien que des doses massives de liberté sont sans effet sur nous, alors que cette même démocratie serait un poison mortel à dose homéopathique dans un pays comme I'U.R.S.S. Mon avis est du reste que la démocratie est, dans le monde entier, condamnée à mort ainsi qu'on le verra dans un délai plus ou moins bref. La simple raison en est que ça ne marche pas et que ce qui ne fonctionne pas doit disparaître... Ce qui le remplacera ? Ce qu'il y a de pire, probablement... »

Mauriac subit patiemment l'étalage de ces propos avant de renoncer: «À ces paroles, en succèdent d'autres où réapparaît le bout de l'oreille du petit loup égaré qu'il fut sous l'occupation. La politique de Vichy n'est plus cette fois glorifiée par lui de façon artificielle, pour l'honneur, mais défendue fanatiquement en vertu d'une adhésion intérieure. Et lorsque je dis : politique de Vichy, j'entends politique des vichystes de sa race, et sans doute même : la sienne seule. J'écoute peu, alors. Ses partis pris m'agacent 
dans la mesure où ils heurtent les miens. »

Les années passent et soudain la nouvelle tombe. «Paris, jeudi 30 mai 1974, écrit Mauriac dans ses souvenirs. Après une disparition, un effacement, un oubli, de plusieurs années, Gaston Bergery est mort, il y a quelques mois, sans que j'en sois ému - ce n'était plus lui, ce n'était plus moi -, sans même, et j'en éprouve du remords, que j'écrive à Bettina. Quelques lignes dans les journaux, alors qu'il eut, pour tant d'entre nous, une telle importance. »

Le long récit de Claude Mauriac s'est achevé. Mais j'en trouve d'autres plus brefs, plus émiettés qui disent encore la place que Bergery a eue pour toute une génération. En 1959, dans son Autocritique, le sociologue Edgar Morin ${ }^{8}$ a raconté son adhésion au frontisme : « J'avais trouvé dans la Flèche un syncrétisme qui ficelait, assez lâchement du reste, les sentiments qui s'embrouillaient en moi : la fraternité antifasciste, la critique libertaire, le pacifisme, le rationalisme sceptique (l'acceptation de perspectives restreintes) de sorte que mon désordre intérieur se sentait à l'aise dans le désordre de La Flèche, tout en y puisant une impression d'ordre. »

Au cours de l'hiver 38-39, Morin avait assisté aux réunions des étudiants frontistes: "Le frontisme m'était apparu comme l'adaptation la plus humaine aux nécessités du siècle. Après septembre 39, les chances frontistes furent exclues, si tant est qu'elles eussent jamais existé. Je me plongeais dans l'étude. »

Le 23 juin 2010, j’ai rencontré Edgar Morin chez sa fille Irène et il m’a parlé de son passé. « Je suis allé souvent à des meetings de Bergery, m'a-t-il dit. J'étais alors inscrit aux jeunesses frontistes. J'étais séduit par l'homme qui avait un discours rigoureux et ne faisait pas de gestes. Quand lui on faisait des compliments, il avait l'air indifférent. Il était sobre, rigoureux, rationnel, convaincant. Pas un orateur à tripes. »

Morin a continué en fouillant dans l'histoire. « J'ai revu Bergery par hasard en 1963 avec Maurice Clavel. Il avait un peu vieilli. Glabre, mince, les traits bien marqués. C'était un caractère. Quand je lui ai dit que j'étais aux jeunesses frontistes, il m’a lancé : “Et nous avions raison!" »

\footnotetext{
${ }^{8}$ Edgar Morin, Autocritique (Paris : Julliard, 1959).
} 
Après cette rencontre, je continuai ma recherche. Elle me conduisait de plus en plus loin. À chaque étape, je trouvais des hommes et leur histoire. Des noms s'ajoutaient à ma liste. Ils étaient avocats, écrivains, étudiants, certains très jeunes, d'autres plus âgés. Je retiens celui de ce préfet de police de Paris qui, en 1968, devra affronter les troubles du printemps. «Avec quelques camarades, raconte Maurice Grimaud ${ }^{9}$, nous avions adopté Gaston Bergery, le brillant fondateur du Pari frontiste qui entendait combattre à la fois le fascisme et le communisme et dont le journal La Flèche dénonçait la toute puissance occulte des trusts. »

Maurice Grimaud après Claude Mauriac, Edgar Morin et tant d'autres.

\section{Photos sépia.}

Ma route continuait. Dans un livre de Jérôme d'Astier ${ }^{10}$, je rencontrai celui que je cherchais. Cet écrivain est le fils d'Emmanuel d'Astier de La Vigerie et de Louba Krassine, la deuxième femme de Gaston Bergery. Dans des pages très émouvantes, Jérôme d'Astier évoque longuement sa mère. «Elle avait seize ans, écrit-il, quand elle a connu un jeune avocat, député socialiste français. II avait un journal qui s'appelait La Flèche. Elle l'a épousé. À vingt ans, elle a eu mon premier frère, le demi. Elle devait assister aux banquets de la circonscription. La Sarthe. Les femmes des notables qui lui parlent des meilleures variétés de haricots verts. Elle ne sait quoi répondre. Elle les remercie de leur compliment sur sa robe Balenciaga. En roulant les $r$. En souriant de tous ses yeux. Elle est une curiosité. La fille d'un sanguinaire, un bolchevique qui tient un couteau entre les dents ${ }^{11}$. »

Le jeune avocat s'appelait Gaston Bergery. Son second mariage avec la fille du premier ambassadeur en France de la nouvelle URSS avait été un magnifique coup de poker qui avait accru considérablement son prestige dans la gauche française. Bergery

\footnotetext{
9 «Du bon emploi des fièvres de mai », entretien de Maurice Grimaud avec Hélène L'Heuillet et Stéphane Thibierge, La célibataire 7 (printemps 2003),133-144.

10 Jérôme d'Astier, Je parlerai de toi à mon ami d'enfance (Paris : Gallimard, 2008).

${ }^{11}$ d'Astier, Je parlerai de toi à mon ami d'enfance, 38.
} 
s'était rendu dans la patrie du communisme. «Elle me dira, raconte d'Astier ${ }^{12}$, qu'elle est retournée en Russie en 1925, avec son premier mari, le jeune député socialiste. Elle était seule dans la loge officielle du Bolchoï, un soir, quand un homme est entré. Elle a reconnu le camarade Staline qui l'a saluée et questionnée très poliment, en la dévisageant. Il avait l'air d'un renard. Sous le rayon X de son regard, elle a répondu. Il demandait des nouvelles de son père, qui était très malade, à Londres. II avait quelque chose de légèrement oppressant. Les yeux. La voix pateline. Une impression désagréable. »

L'itinéraire familial me parut une piste intéressante. Alors je cherchai dans l'annuaire et je finis par mettre la main sur le numéro de téléphone d'un des petits-fils. J'appelai Benjamin Bergery et pris rendez-vous avec lui à Paris pour le lundi 19 octobre 2009. Nous avions convenu de nous retrouver à 16 heures à la station Stalingrad. Mais dans le métro, mon portable sonna. II me dit d'aller jusqu'à Jaurès. En haut des escaliers, je trouvai un homme de mon âge, quelques années de plus peut-être, avec un air d'artiste triste. II me raconta qu'il travaillait dans la photo et le cinéma. Installés sur une terrasse face au canal, nous regardâmes le mouvement. Ses mots étaient rares. Il y eut beaucoup de silences. Son grand-père ? Il ne l'avait vu que deux ou trois fois et ils n'avaient rien reçu de lui. Pas de papiers, de lettres, d’objets. Rien! Soudain il sortit un appareil et fit des photos. Des photos de moi ! Mais il refusa que je le photographie. Nous marchâmes le long du canal. Il faisait froid malgré le soleil. Alors nous entrâmes dans un autre café. Il me parla de la guerre. «Qu'aurions-nous fait ? dit-il. Mais pourquoi un homme aussi intelligent que Bergery a-t-il pu se tromper? »

Je le quittai et retournai à ma recherche. Au printemps de cette année-là, j'avais publié une fiction $^{13}$ où j'avais placé un certain Gaston B. B. comme Bergery! Pour composer le personnage, je m'étais servi de tout ce que j'avais accumulé sur lui. Le 3 juillet 2009, je reçus ce courriel que je cite dans son intégralité.

«Monsieur,

J'ai lu avec un grand intérêt votre roman Trois coupes de champagne, en particulier

\footnotetext{
${ }^{12}$ d'Astier, Je parlerai de toi à mon ami d'enfance,137.

${ }^{13}$ Yves Pourcher, Trois coupes de champagne (Paris : Grasset, 2009).
} 
le chapitre "Moscou" dans lequel vous racontez le retour en France du personnel de I'Ambassade après la déclaration de guerre de I'Allemagne à I'URSS. En 1941, j'ai fait partie de ce personnel en qualité de secrétaire d'Eirik Labonne ${ }^{14}$, puis de "Gaston B." et, en mai et juin, j'ai dû remplacer le chiffreur parti en vacances. Les "équipes de déchiffreurs" auxquelles vous faites allusion se réduisaient à ma modeste personne !

Ayant effectué avec tout le personnel diplomatique le voyage - très mouvementé ! - dont vous donnez un aperçu, j'ai certainement connu la personne qui vous en a fait le récit. Serait-il indiscret de vous demander de bien vouloir me donner le nom et l'adresse de cette personne ? Je serais très heureuse...s'il en est encore temps...de pouvoir la joindre pour évoquer avec elle ces lointains souvenirs....

En vous remerciant très vivement à l'avance de la suite qu'il vous plaira de donner à ce message, je vous prie, Monsieur, de croire à mes sentiments distingués.

Denise HERSANT, née DUPONT (c'est le nom que je portais en 1941). »

Je la rencontrai le 21 septembre 2009 à Bayonne où elle vit. Ce moment fut exceptionnel. Pendant des heures, j'écoutai passionné le récit qu'elle et son mari me firent de ces années. Elle me décrivit Bergery : «Un personnage très déconcertant avec un regard très froid. Mais il n'était pas du tout froid avec moi. Il a même été amusant. II n'était pas diplomate pour deux sous, faisait des boutades. » Elle conclut avec sa fin : «En 1974, la messe d'enterrement a eu lieu à Sainte-Clotilde. L'église était pleine. J'ai rencontré des camarades de Moscou que j’avais perdu de vue. »

Qui donc en définitive était cet homme appelé Gaston Bergery? J'avais jusqu'à présent accumulé les références livresques et cité quelques témoignages qui parfois se contredisaient. Comme si les souvenirs brouillaient la vérité et effaçaient le passé. Mais ce besoin artificiel de vie m'avait éloigné de mes premiers pas. Pour mettre des couleurs sur la photo du personnage, j'avais délaissé les chemises dans lesquelles j'avais méticuleusement rangé mes documents d'archives. Trop sombres ! avais-je alors pensé, trop coupées et dispersées. Pourtant une nouvelle fois, je tendis la main et les saisis. J'exhumai des pages qui venaient de lieux différents. Dès lors, ma recherche s'apparenta

\footnotetext{
${ }^{14}$ Eirik Labonne, ambassadeur de France à Moscou (12 juin 1940-avril 1941).
} 
à un voyage. Je prenais l'avion, le train, le métro. À chaque porte où je frappais, je trouvais des moments de vie. Pour bien me faire comprendre, je vais indiquer précisément les adresses et les institutions. Car chacune d'entre elles conserve un moment de cette vie.

\section{Château de Vincennes.}

J'avais demandé un dossier d'officier"15. «BERGERY Gaston-Franck » était-il écrit sur la couverture du dossier. Je notai quelques informations. "Jeune soldat appelé bon service armé de la classe 1914 de la subdivision de la Seine ». Son signalement : cheveux blonds, yeux gris-bleu, visage ovale, taille $1 \mathrm{~m} 75$.

Le 2 septembre 1914, Bergery fut incorporé au $117^{\mathrm{e}}$ régiment d'infanterie. II partit aux armées le 28 octobre. Nommé caporal le $1^{\text {er }}$ décembre, il devint sergent le 27 du même mois puis aspirant le 21 février 1915. Le 3 mars 1915, il fut cité à l’ordre du Corps d'armée : «A fait preuve d'une énergie et d'une vigueur admirable en entraînant sa troupe à l'assaut pendant le jour et la contre-attaque de la nuit 23 février. »

Bergery fut blessé le 29 septembre 1915 à l'Epine de Videgrange en Champagne: contusions multiples par éclats d'obus. Le 10 avril 1916, il entra à l'hôpital de Bordeaux d'où il sortit le 14. Il fut alors évacué sur le dépôt de convalescents de la caserne Faucher pour troubles cérébraux et oculaires par éclatement d’obus.

Le 17 juin 1916, Bergery partit au Havre, affecté à la mission militaire française auprès de l'armée britannique. Il servit comme officier interprète du 13 septembre 1918 au 12 février 1919 date à laquelle il fut mis la disposition du ministre pour le service de la Conférence de la paix. Sa mission s’acheva le 30 août 1919. Le lendemain, il fut démobilisé.

Bergery avait été nommé sous-lieutenant le 13 septembre 1918. Il deviendra lieutenant le 8 mai 1924 et capitaine le 25 décembre 1933. Toujours bien noté, Bergery ne sera pas mobilisé en 1939.

Tout me semblait parfait. Bon soldat, sous-officier puis officier distingué, noté

\footnotetext{
${ }^{15}$ Service historique de l'armée de Terre 8Ye 109716
} 
comme courageux, cultivé et efficace. Mais soudain le contenu du dossier changeait de sens. Une liasse de notes et de rapports dénonçait l'activité pacifiste et les options politiques de Bergery. "Prépare la révolte contre le gouvernement et la guerre civile », était-il précisé. Des enquêtes avaient été menées. Bergery fut même menacé d'arrestation. Le 17 mai 1934, le général de division Pretelat avait écrit à M. le Maréchal de France, ministre de la Guerre: "J'ai l'honneur en conséquence de proposer que cet officier de réserve soit traduit devant un Conseil d'enquête aux fins de révocation pour faute grave contre la discipline. »

Le maréchal de France s’appelait Philippe Pétain.

\section{Quai d'Orsay.}

Aux archives du ministère des Affaires étrangères, je trouvai le dossier personnel de Gaston Bergery ambassadeur de la France de Vichy en poste à Moscou le 5 avril 1941, puis à Ankara le 16 juin 1942. Étaient indiqués ses entrées en fonction, son salaire et l'état des lieux des bâtiments qu'il avait occupés. Chaque jour, "son excellence Bergery » recevait, écrivait et signait. Toutes les lettres et les télégrammes étaient précieusement conservés. J'en retiens quelques-uns.

Télégramme à l'arrivée $n^{\circ} 749$, envoyé le 9 juillet 1942 à 18 h50 et reçu à 23 h50 :

« Je suis arrivé ce matin à Istanbul où j’ai été salué à la gare par notre chargé d'affaires, le consul général et tous les membres représentatifs de la colonie. Je pars demain soir vendredi pour Ankara où je prendrai immédiatement le service. Bergery. »

Autre message envoyé d'Istanbul, le 2 septembre 1942 :

« M. Von Papen m'ayant invité la semaine dernière à déjeuner à Therapia dans l'intimité, je lui ai rendu aujourd’hui cette politesse. II a déjeuné au Palais de France avec Madame von Papen et ses trois filles. Il est impossible de montrer plus de considération et de sympathie qu'il n'en a montré. II m'a parlé des choses de France dans des termes qui m’ont ému. Je serais tenté de croire que cette amabilité était exceptionnelle si M. von Papen n'avait la réputation d'être un homme toujours exceptionnellement aimable. Après 
déjeuner, nous nous sommes retirés dans mes bureaux et nous avons parlé en tête à tête de toutes les questions dans lesquelles, en Turquie, les intérêts de la France et de l'Allemagne sont simultanément engagés. Sur toutes ces questions, nous avons réalisé un accord complet. »

Ernst Von Papen est alors l'ambassadeur d'Allemagne à Ankara. Représentant de la droite catholique dans son pays, il avait permis l'arrivée des nazis au pouvoir.

Depuis des mois, la guerre se joue à l'Est, au milieu de l'immense URSS. Le sort de l'Europe et du monde hésite. Au sein de l'ambassade, certains font des choix et Bergery réagit et frappe. II révoque des collaborateurs. Le 2 septembre 1942, il écrit au ministère pour proposer des retraits de nationalité. Les noms s'alignent sur les lettres avec les motifs des sanctions proposées :

« JOUVE Géraud, chef des Gaullistes de Turquie, a ses bureaux dans l'ambassade d'Angleterre, délivre des visas pour la Turquie. Ancien correspondant de l'Agence Havas à Berlin, agrégé d'allemand.

MANAC'H Étienne-Manoël, professeur adjoint de l'enseignement secondaire à Galata-Saray- adjoint de Jouve.

MOUSSAT Édouard-Constantin, principal rédacteur du bulletin gaulliste de Turquie. »

Quelques jours plus tard, sur du papier à lettres à en-tête de l'Ambassade de France en Turquie, Bergery écrit : «Je jure fidélité à la personne du Chef de l’État et je m'engage à exercer ma charge pour le bien de l'État selon les lois de l'honneur et de la probité $^{16}$. »

Le même matin, il avait complété un imprimé du ministère des Affaires Étrangères: «Nom: Bergery. Prénom: Gaston. Je soussigné, déclare sous la foi du serment, n'avoir jamais appartenu, à quelque titre que ce soit, à l'une des sociétés suivantes: Grand Orient de France, Grande Loge de France, Grande Loge Nationale Indépendante, Ordre Mixte International du Droit Humain, Société Théosophique, Grand

\footnotetext{
${ }^{16}$ Istanbul le 22 septembre 1942. La lettre est signée Gaston Bergery, Ambassadeur de France.
} 
Prieuré des Gaules, à l'une quelconque des filiales desdites sociétés, ou à toute autre société visée par la loi du 13 août 1940 et m'engage sur l'honneur à ne jamais en faire partie, au cas où elles viendraient à se reconstituer directement ou non. »

Un ensemble intitulé Papiers Bergery, «la collection de l'ambassadeur», rassemble les télégrammes expédiés et reçus par le poste d'Ankara. Vingt-sept photos sur la vie à l'ambassade complète le tout. On y voit Bergery en costume blanc et son épouse en robe élégante recevant, visitant, montant sur un yacht. Les conservateurs, qui présentent le fonds, disent qu'on ne sait pas dans quelles circonstances ces papiers ont été donnés aux Archives diplomatiques. Quand Bergery avait-il exactement remis ces documents?

Je lisais patiemment tous les feuillets en notant les nuances qui s'établissaient dans les relations. Elles me semblaient traversés par les sous-entendus, les atermoiements ou les reproches. Pourtant une même politique de faillite et de compromission s'appliquait tant bien que mal. Le 15 septembre 1942 à 11h10, le chef du gouvernement de Vichy avait écrit : "Je me réfère à votre télégramme 1202-12. Je vous fais pleinement confiance dans la tâche d'épuration et d'union que vous assumez et dont je connais les difficultés. Je fais de mon côté le nécessaire pour hâter les formalités de retrait de nationalité demandées par votre télégramme 1134-41. Pierre Laval. »

Bergery appliquait, au moins en apparence, les nouvelles lois, même les plus sordides. Le 12 janvier 1943, il avait par télégramme demandé des précisions : «Je serais obligé au Département de bien vouloir me faire savoir si la femme juive française d'un mari aryen et français peut, dans les conditions actuelles, recevoir un visa de sortie de France pour rejoindre son mari à l'étranger. Je ne pose cette question qu'en principe et sous réserve bien entendu des cas d'espèce. »

Tous ensemble ces documents rendent compte de la compromission, de l'ambiguïté et de la montée des périls. Bergery était un homme de Vichy. Mais il savait aussi voir et écouter. Ensuite il rapportait la teneur de ses entretiens. Dans ses lettres, je relevais des nuances, des évolutions et des glissements. Où passe la frontière entre la fidélité à cet ignoble régime et la prise de distance? L'archive révèle parfois un lent 
cheminement. Ainsi le 21 juillet 1944 arriva à l'ambassade un mot qui traduisait le mécontentement ${ }^{17}:$ «J'ai bien reçu la lettre que vous avez adressée le 11 juin à $M$. Selim Saper pour protester contre l'article du "Yeni Sabah" du 9 juin. J'ai lu l'article. Il me vise et m'injurie autant que le Maréchal. Vous avez eu raison de protester. Je regrette seulement que vous m'ayez omis dans votre protestation. Si personnellement je méprise les insultes, je vous rappelle qu'en France la législation punit les injures visant les Ministres des Affaires Étrangères autant que les Chefs de l'État. »

La lettre était signée de Pierre Laval.

La fin de la guerre approchait. Après la libération de la France, Bergery quitta l'ambassade tout en restant prudemment en Turquie. De leur refuge, son épouse Bettina envoya un courrier à une amie : «Je vous ai écrit lorsque nous avions quitté en avril, le Park-Hotel pour nous fixer sur cette côte de l'Asie. Depuis nous attendons dans une gentille maisonnette avec un grand jardin et une crique avec un petit voilier, nous attendons un bateau plus grand qui nous ramènera chez nous. Quelle longue attente depuis le mois de septembre. Gaston a des malles pleines de documents de toutes ses missions. Il doit les prendre avec lui, car il ne peut pas les laisser lire par les officiers de la douane et ne peut pas risquer, non plus de les voir confisqués en route. C'est pourquoi, nous attendons un bateau direct pour la France, français ou turc. Jusqu'ici il n'y avait qu'un cargo qui n’a pas pris de voyageur. Il y a quelques mois, Gaston a écrit au Général de Gaulle, et expliqué sa situation. Il a demandé le moyen de ramener ses papiers comme bagages personnels aussitôt que possible. Jusqu'ici, pas de réponse ${ }^{18}$. »

Un bateau était passé. Les Bergery étaient rentrés en France.

\section{Rue des archives.}

Dans les documents du procès conservés aux archives nationales ${ }^{19}$, je trouvai d'abord une notice individuelle de la direction générale de la sûreté nationale. Un homme

\footnotetext{
17 Il avait été envoyé de Vichy le 14 juillet 1944.

${ }^{18}$ Lettre de Bettina Bergery à Miss Alice de Samar, 530, Park av. New York le 5 juin 1945

${ }^{19}$ Archives nationales F7/15326.
} 
était présenté. Sa taille, $1 \mathrm{~m}$ 72, la barbe rasée. Il avait été avocat, homme politique puis ambassadeur. Sa situation militaire: capitaine de réserve. Sur mandat de M. Marchat, juge d'instruction à la Cour de Justice de la Seine, Gaston Bergery avait été arrêté le 25 octobre 1945 et aussitôt inculpé d'intelligence avec l'ennemi.

Les interrogatoires avaient commencé le 26 octobre. Ils sont tapés sur des pelures annotées et soulignées. Bergery revient sur sa jeunesse, sa carrière politique, ses ambassades. Il accumule les détails sur ses actes, ses fréquentations et plaide sans cesse pour sa cause.

Les auditions s'étaient succédé. Je retiens celle qui a eu lieu le 29 octobre 1945 en énumérant les questions posées :

«Avez-vous effectué des voyages à l'étranger notamment en Allemagne, avant la guerre et sous l'Occupation ? »

«Comment et dans quelles circonstances êtes-vous entré en relations suivies avec le Maréchal Pétain ?»

«Avez-vous assisté à une réception organisée le 8 décembre 1941, par Goering, à Paris ? "

«À titre d'ambassadeur de France à Ankara, avez-vous été appelé à demander des sanctions contre des membres de la colonie française. »

Les réponses suivent et cela se termine toujours par la même formule: «lecture faite, persiste et signe, le commissaire de police (... )».

La justice fouillait le passé de Bergery. Elle cherchait à fixer son rôle pendant l'Occupation. Il reconnaissait avoir rencontré Abetz à Paris sur les instructions du maréchal en ne regrettant pas son attitude. Les jours suivants, l'interrogatoire s'était poursuivi. Je tournais les pages de ces archives d'un procès politique. Enfin le 12 février 1949 à 22 heures, la 1ère section de la Cour de justice de la Seine avait rendu son jugement dont je rapporte les principales conclusions :

« $1^{\text {ère }}$ question. Bergery Gaston, accusé ici présent, est-il coupable d’avoir en France et à l'étranger, de 1940 à 1944, en tous cas entre le 16 juin 1940 et la date de la libération, 
en temps de guerre, étant Français, entretenu des intelligences avec une puissance étrangère ou avec ses agents, en vue de favoriser les entreprises de toutes natures de cette puissance étrangère contre la France, ou l'une quelconque des nations alliées en guerre avec les puissances de l’axe. »

Réponse : non à la majorité.

Suivent ensuite d'autres compléments : il n'avait pas nui à la défense nationale ; il n'était pas coupable d'indignité nationale. En conséquence, Gaston Bergery bénéficia d'une relaxe pure et simple ${ }^{20}$.

Après ce verdict et pour aller plus loin, je parcourais des inventaires. Je cherchais dans diverses séries. Sur un microfilm, je lus le journal de Marcel Déat ${ }^{21}$ qui avait été l'un des responsables les plus acharnés de la politique de la Collaboration. Le nom de Bergery revenait à de multiples reprises. Je saisissais ses apparitions comme des flashes, des jaillissements de lumière au milieu de mes sombres archives. Ils témoignaient d'un moment, d'une attitude ou d'une pensée. La silhouette de Bergery s'animait. Ses lèvres bougeaient, son corps avançait. Je le voyais, je l'entendais. Après la rupture des relations diplomatiques avec l'URSS conséquence de la déclaration de guerre de l'Allemagne contre ce pays, il était rentré en France. « Jeudi 20 novembre 1941, écrit Déat. (...) Arrivée de Bergery : propos intéressants sur l'armée de robots, sur le massacre des élites, sur la terreur de Staline devant la guerre, sur les incidents pittoresques de la dernière semaine avant l'offensive allemande, sur le voyage de retour et l'accident de chemin de fer. »

Quelques jours plus tard, Goering était reçu à Paris et Déat raconte : «Mardi 2 décembre 1941 (...) Retour à la maison. On se prépare à la grande réception en enfilant le smoking. À gh15 nous partons dans la voiture de L'Oeuvre ${ }^{22}$ avec Prieur. Soirée sensiblement comme celle du lundi soir, avec plus de monde et d'uniformes. Arrivée du maréchal, présentation. On bavarde dans tous les coins. Buffet somptueux. Laval parle un peu avec Goering. Abetz veut aussi que je parle avec le Maréchal, mais il est trop tard. Et

\footnotetext{
${ }^{20}$ Archives nationale AL/5299.

${ }^{21}$ Archives nationales F7 15342.

${ }^{22}$ L'Oeuvre, journal dirigé depuis juillet 1940 par Marcel Déat.
} 
d'ailleurs, pour dire quoi? Vu Bergery, en uniforme d'ambassadeur, avec Bettina. Vu Schueller, Mme Fontenoy, Mme Lacombe, Paul Morand, du Jonchet, Arletty, les de Chambrun, Mgr Mayol de Lupé, etc, etc, et naturellement une foule d'allemands civils et militaires. Nous rentrons à pied au clair de lune et Prieur s'appuie la traversée de la moitié de Paris. »

Bergery avait paradé en grande tenue à l'ambassade d'Allemagne. Il aimait tellement cela.

Je sortis de ce lieu avec le doute et le dégoût dans la tête. Jusqu'où Bergery était-il allé dans la compromission? Et puis je m'interrogeais pour savoir si je n'avais rien omis. Étais-je bien passé partout? N’avais-je rien laissé de côté ?

\section{Nouvelles pistes.}

Je me rendis aux archives de la préfecture de police de Paris où je ne trouvai rien. Bergery n'avait ni fiche ni dossier. Alors ne sachant plus quel chemin prendre, je me tournai vers mes propres archives ouvrant les cartons dans lesquels j'avais rangé les notes que j'avais prises entre 1994 et 1997 dans le grand appartement de Josée de Chambrun $^{23}$, la fille unique de Pierre Laval. Je relus ses agendas intimes ${ }^{24}$. Les Chambrun et les Bergery s'étaient beaucoup reçus pendant l'Occupation. Le 15 octobre 1945, Laval avait été fusillé et, les années suivantes, sa fille et son gendre avaient constitué autour d'eux une sorte de chapelle des fidèles de Vichy. Pourtant les rancoeurs étaient nombreuses dans ce petit monde de nostalgiques et d'aigris. En plein procès, l'avocat René de Chambrun avait écrit à Bergery pour lui dire en termes nuancés son mécontentement :

« Mon cher Gaston,

J'étais à l'audience d'aujourd'hui et j'ai approuvé et apprécié beaucoup de choses que tu as dites ; mais permets-moi de te faire remarquer très affectueusement :

\footnotetext{
${ }^{23}$ Au 6 bis place du Palais-Bourbon à Paris.

${ }^{24}$ Yves Pourcher, Pierre Laval vu par sa fille (Paris: Le cherche midi, 2002) et Yves Pourcher, «Laval museum ", Historical reflections 38, 1 (2012), 105-125.
} 
$1^{\circ}$ - que mon beau-père n’a jamais aspiré faire la guerre à la Grande-Bretagne. II te suffira pour t'en convaincre de lire le texte de cette lettre que Josée a récemment écrite à M. Paul Baudoin ${ }^{25}$.

$2^{\circ}$ - que “l'alternative” pour mon beau-père de t'envoyer à Ankara ou de te mettre “en tôle” ne s'est jamais posée ? C'est à Josée que tu es venu demander, en avril 1942, le poste d'Ankara. C'est moi qui ai été ton ambassadeur pour l'obtenir et tu es parti là-bas je le jure sur la mémoire de mon beau-père - avec son entière confiance. Bien affectueusement à toi ${ }^{26}$. »

La réponse n'avait pas tardé. " 1 heure, avait noté Josée dans son carnet Hermès le 14 février 1949, Bergery pour me raconter son procès, mettre les choses au point et faire des excuses. » Les rapports s'étaient apaisés. À cette époque, les Bergery fréquentaient un très sombre personnage, Oswald Mosley, qui, les années précédant la guerre, avait été le leader des fascistes anglais. "Gentil déjeuner chez les Mosley hier avec les éternels Bergery ", écrivait Josée à son mari le 19 avril 1956. Leurs relations étaient aussi mondaines.

J'arpentais les bibliothèques. Dans un gros livre intitulé Journal de France qui rassemble les agendas d'Angelo Guiseppe Roncalli ${ }^{27}$, nonce apostolique à Paris, je trouvai le nom de Bergery. Les deux hommes s'étaient connus en Turquie et s'étaient appréciés. Le 27 avril 1946 précisément, le nonce avait reçu l'avocat de Bergery qui avait recueilli son témoignage en sa faveur. Et après sa libération, les 30 avril et $1^{\mathrm{er}}$ juin 1948, Gaston Bergery était allé vite solliciter le prélat. "L'ambassadeur Bergery, avait noté Roncalli dans son carnet, qui m’a beaucoup pressé de l'aider. » Bergery avait obtenu une nouvelle audience le 23 octobre. "Oh! comme le monde politique est traître, avait écrit le nonce. II ne pardonne à personne. Pour moi Bergery est une âme à aider. » Les mois et les années suivantes, les deux hommes avaient continué à se fréquenter.

\footnotetext{
${ }^{25}$ Paul Baudoin, ministre des Affaires Étrangères du 16 juin au 29 octobre 1940.

${ }^{26}$ Archives privées Fondation Josée et René de Chambrun, lettre du 7 février 1949.

${ }^{27}$ Angello Giussepe Roncalli, Journal de France. I 1945-1948 (Paris : Cerf, 2006). Mgr Roncalli deviendra pape sous le nom de Jean XXIII en 1958.
} 
Sur une autre étagère, je trouvai le Journal inutile de l'écrivain Paul Morand ${ }^{28}$, autre ambassadeur de Vichy. Le 15 février 1974, il avait noté la fin : « Mort de Bergery, à 80 ans. Il était apoplectique depuis 10 ans. Fin lamentable, comme presque toutes. Je l'avais connu en 1924. Il avait conquis Herriot ${ }^{29}$. Ses réceptions dans un studio décoré et meublé à la mort de Ruhlmann, vers 1925. Puis dans le monde. J'avais rencontré Bettina à New York, avant son mariage, en 27, encore mannequin. Puis la période fasciste de La Flèche, en 36-40. Retrouvé à Vichy en 42. Le premier à me dire: "Les Russes ont gagné, les Allemands sont perdus." Ambassadeur à Ankara, il était venu avec Bettina passer quelques jours chez nous, à Bucarest, en 1944. »

Je quittai les bibliothèques et les librairies. Pourtant une question me taraudait toujours l'esprit. Étais-je allé jusqu'au bout de cette histoire? En réalité, je savais bien qu'il existait d'autres documents, lointains, presque inaccessibles. L'historien Philippe Burrin les avait consultés. Comme quelques autres individus liés au régime de Vichy et à la Collaboration, Gaston Bergery avait donné avant sa mort ses archives personnelles à la fondation Hoover située à Stanford ${ }^{30}$. J'écrivis alors à cette adresse. Après plusieurs échanges, une responsable me transmit un jeu de photocopies. Cela m'aida à attendre et, en même temps, aiguisa ma curiosité. L'inventaire indiquait brièvement le contenu: correspondances, télégrammes, rapports, discours et écrits personnels. Au mois de mai 2011, j'arrivai enfin à la petite gare de Palo Alto, puis je marchai vers la tour Hoover. J'entrai dans le bureau de Linda Bernard, la conservatrice. Elle me présenta la fondation, puis me parla du fonds Bergery qui avait été méticuleusement classé et rangé. II était ouvert. Mais je devais venir travailler sur place. Quelques heures plus tard, je repris mon train vers San Francisco en me promettant de revenir vite.

Je rentrai en France et, quelques semaines plus tard comme je le faisais souvent, je tapai le nom de Bergery sur mon clavier. Je découvris alors que l'université de Yale avait

\footnotetext{
${ }^{28}$ Paul Morand, Journal inutile. 1973-1976 (Paris : Gallimard, 2001), $190-191$.

${ }^{29}$ Gaston Bergery avait été chef de cabinet d'Edouard Herriot au ministère des Affaires Étrangères (19241925).

30 René de Chambrun avait lancé le mouvement. Sous l'égide de cette fondation, il avait rassemblé les témoignages des collaborateurs de son beau-père, Pierre Laval.
} 
acquis, en 2002, les papiers personnels de Bettina ${ }^{31}$. L'inventaire indiquait trente cartons comprenant ses agendas, des livres, des lettres et des notes. Sur le site, je la voyais en photo vêtue d'une magnifique robe de soirée ou en tenue de ski, et lui, Gaston, portant l'uniforme d'ambassadeur. Un nouveau monde s'ouvrait qui m’apparaissait inaccessible. Aussitôt, et comme souvent au cours de mes aventures dans les archives, la figure du labyrinthe m'apparaissait. De tous côtés, elle lançait ses routes, ses chemins, ses sentiers qui se croisaient et se superposaient. Comment devais-je faire pour les suivre ? Étais-je tout simplement pris dans le piège ordinaire de la biographie ou cette vie-là, celle de Gaston Bergery, me jouait-elle un mauvais tour? Pourtant je m'acharnais en achetant des lots d'exemplaires de La Flèche, le journal de Bergery que je lisais patiemment. J'y trouvais ses discours, ses déclarations politiques et ses articles polémiques. L'actualité politique de ces années trente défilait à travers ces pages. Sur des sites spécialisés, je saisissais aussi des textes parlant de lui et des photos. Mais cela ne me suffisait pas.

\section{Labyrinthe.}

Depuis plusieurs mois, j'étais entré en relation avec un journaliste, écrivain et homme de télévision : Jean-José Marchand ${ }^{32}$. Je l'avais contacté un peu par hasard parce qu'au même moment je cherchais aussi sur un autre personnage, un individu tout aussi inaccessible que Bergery ${ }^{33}$. Les archives municipales d'Arcachon m'avaient donné un numéro de téléphone que j’avais composé très vite. Jean-José Marchand m’avait écouté en me demandant de lui envoyer quelques-uns de mes livres. Depuis je l'appelais régulièrement. II me parlait de l'entre-deux guerres, de la période de l'Occupation et me guidait dans mes recherches.

\footnotetext{
${ }^{31}$ Yale University Library, Beinecke Rare Book and Manuscript Library. General Collection of Modern Books and Manuscripts. Guide to the Bettina Bergery Papers GEN MSS 844 by Karen Spicher, August 2012

32 Jean-José Marchand (1920-2011). On peut lire de lui, récemment publiés, Jean-José Marchand, Écrits critiques (Paris : Éditions du Félin-Éditions Claire Paulhan, 2012), 5 volumes sous coffret (dont 1 vol. d'index), 2910 pages.

33 II s'agissait de Jean Fontenoy, personnage sulfureux, ancien journaliste à l'agence Havas qui sombra et mourut dans la Collaboration. Un livre récent a fait le point sur sa vie et son parcours : Gérard Guégan, Fontenoy ne reviendra plus (Paris : Stock, 2011).
} 
Jean-José Marchand savait tout. Quand il me parlait de son âge, «presque centenaire » disait-il en exagérant, c'était pour ajouter aussitôt : «Le seul mérite d'être vieux, c'est d'avoir vu ce qu'ont fait les autres ${ }^{34}$. » II connaissait parfaitement la vie de Bergery. "Un personnage tout à fait remarquable qui avait une vision », commentait-il. "Et qui avait beaucoup de goût », ajoutait-il. Jean-José Marchand avait connu son fils, Jean-François, scénariste de métier, « un très joli garçon qui avait épousé une très belle Américaine ». Mais sur son père, Marchand avait eu des mots définitifs qui restent encore aujourd'hui très étranges: "L'histoire l'a trompé comme une mauvaise maîtresse ${ }^{35}$. » Était-ce la conclusion de cette histoire? Un soir pourtant, je repris le livre de Jérôme d'Astier. "Le passé est comme un collier de perles brisé », avait-il écrit. Mais ces perles s'étaient-elles dispersées? Contre vents et marées avaient-elles réussi à garder leurs couleurs et leurs secrets? Et moi, sur cette route, ne m’étais-je pas égaré ?

Pourtant le soir ${ }^{36}$ même où, à Toulouse, je présentai ce Dossier Bergery devant un petit groupe de collègues, était annoncé sur les murs de la ville un grand meeting politique. La campagne pour les élections présidentielles animait depuis des mois le débat public. Sur la grande place du Capitole située au cœur de la ville, j’allai écouter Jean-Luc Mélanchon candidat du Front de Gauche. «Dans cette saison des tempêtes qui déferlent sur le monde, avait-il écrit dans son tract, c'est l'heure des caractères. Donnons le meilleur de nous-mêmes pour faire face. Nous sommes capables de surmonter toutes les difficultés. Le bonheur commun est possible. » Son texte continuait ensuite avec un appel au peuple souverain. «Vienne le temps des cerises et des jours heureux ! » concluait-il.

En avançant vers la place, je vis la foule ${ }^{37}$ qui peu à peu se formait. Certains tenaient dans leurs mains des drapeaux rouges. Le meeting était en plein air. Les discours commencèrent. Des orateurs chauffèrent la place. Puis il arriva sur l'estrade en costume noir, chemise blanche et cravate rouge. Il commença en saluant Jean Jaurès, un fils de ce pays. Puis pendant trente minutes, Jean-Luc Mélanchon appela à la révolte contre les

\footnotetext{
${ }^{34}$ Conversation téléphonique de jeudi 10 septembre 2009.

${ }^{35}$ Conversation téléphonique du 22 septembre 2009.

${ }^{36}$ Jeudi 5 avril 2012.

3770000 personnes selon les organisateurs.
} 
puissants, les riches criant aussi contre le président sortant. L'Internationale, puis la Marseillaise retentirent. Les gens se dispersèrent sous la pluie qui s'était mise à tomber ${ }^{38}$. Je m’éloignai avec ces mots et ces images qui résonnaient dans ma tête et mettaient un point final à cette interminable recherche. Hier un Front commun, avec un homme nommé Bergery, et aujourd'hui, un Front de gauche rassemblant les communistes et des proches, et ce nouveau leader en cravate rouge. Alors je songeai à tous ces hommes qui, dans les plis de l'histoire, cherchent leur destin ouvrant grand leurs bras vers la chance qu'ils appellent et croient voir venir.

\footnotetext{
${ }^{38}$ Le 6 mai 2012, jour du premier tour des élections présidentielles, Jean-Luc Mélenchon obtient 3984822 voix soit $11,10 \%$ des suffrages exprimés.
} 